\title{
The Importance of Emotional Intelligence When Leading in a Time of Crisis
}

\author{
Molly A Kantor, MD*, Sarah K Apgar, MD, Armond M Esmaili, MD, Adeena Khan, MD, Bradley Monash, MD, Bradley A Sharpe, MD \\ Division of Hospital Medicine, University of California, San Francisco, San Francisco, California.
}

cou he coronavirus disease of 2019 (COVID-19) pandemic has created innumerable challenges on scales both global and personal while straining health systems and their personnel. Hospitalists and hospital medicine groups are experiencing unique burdens as they confront the pandemic on the frontlines. Hospital medicine groups are being challenged by the rapid operational changes necessary in preparing for and caring for patients with COVID-19. These challenges include drafting new diagnostic and management algorithms, establishing and enacting policies on personal protective equipment (PPE) and patient and provider testing, modifying staffing protocols including deploying staff to new roles or integrating non-hospitalists into hospital medicine roles, and developing capacity for patient surges ${ }^{1}$-all in the setting of uncertainty about how the pandemic may affect individual hospitals or health systems and how long these repercussions may last. In this perspective, we describe key lessons we have learned in leading our hospital medicine group during the COVID-19 pandemic: how to apply emotional intelligence to proactively address the emotional effects of the crisis.

\section{LEARNING FROM EARLY MISSTEPS}

In the early days of the COVID-19 pandemic, the evolving knowledge of the disease process, changing national and local public health guidelines, and instability of the PPE supply chain necessitated rapid change. This pace no longer allowed for our typical time frame of weeks to months for implementation of large-scale operational changes; instead, it demanded adaptation in hours to days. We operated under a strategy of developing new workflows and policies that were logical and reflected the best available information at the time.

For instance, our hospital medicine service cared for some of the earliest-identified COVID-19 patients in the United States in early February 2020. Our initial operational plan for caring for patients with COVID-19 involved grouping these patients on a limited number of direct-care hospitalist teams. The advantages of this approach, which benefitted from low numbers of initial patients, were clear: consolidation of clinical and operational knowledge (including optimal PPE practices) in a few individuals, streamlining communication with infectious diseases specialists and public health departments, and requiring change on

*Corresponding Author: Molly A Kantor, MD; Email: Molly.Kantor@ucsf.edu; Twitter: @dr_kantor.

Published online first August 19, 2020.

Find additional supporting information in the online version of this article.

Received: May 13, 2020; Revised: June 8, 2020; Accepted: June 8, 2020

(c) 2020 Society of Hospital Medicine DOI 10.12788/jhm.3484 only a couple of teams while allowing others to continue their usual workflow. However, we soon learned that providers caring for COVID-19 patients were experiencing an onslaught of negative emotions: fear of contracting the virus themselves or carrying it home to infect loved ones, anxiety of not understanding the clinical disease or having treatments to offer, resentment at having been randomly assigned to the team that would care for these patients, and loneliness of being a sole provider experiencing these emotions. We found ourselves in the position of managing these emotional responses reactively.

\section{APPLYING EMOTIONAL INTELLIGENCE TO LEADING IN A CRISIS}

To reduce the distress experienced by our hospitalists and to lead more effectively, we realized the need to proactively address the emotional effects that the pandemic was having. Several authors who have written about valuable leadership lessons during this time have noted the importance of acknowledging the emotional tolls of such a crisis and creating venues for hospitalists to share their experiences. ${ }^{1-4}$ However, solely adding "wellness" as a checklist item for leaders to address fails to capture the nuances of the complex human emotions that hospitalists may endure at this time and how these emotions influence frontline hospitalists' responses to operational changes. It is critically important for hospital medicine leaders to employ emotional intelligence, defined as "the ability to monitor one's own and others' feelings and emotions, to discriminate among them and to use this information to guide one's thinking and actions." ${ }^{5-7}$ Integrating emotional intelligence allows hospital medicine leaders to anticipate, identify, articulate, and manage the emotional responses to necessary changes and stresses that occur during a crisis such as the COVID-19 pandemic.

As we applied principles of emotional intelligence to our leadership response to the COVID crisis, we found the following seven techniques effective (Appendix Table):

1. ASK. Leaders should ask individual hospitalists "How are you feeling?" instead of "How are you doing?" or "How can I help?" This question may feel too intimate for some, or leaders may worry that the question feels patronizing; however, in our experience, hospitalists respond positively to this prompt, welcome the opportunity to communicate their feelings, and value being heard. Moreover, when hospitalists feel overwhelmed, they may not be able to determine what help they do or do not need. By understanding the emotions of frontline hospitalists, leaders may be better able to address those emotions directly, find solutions to problems, and anticipate reactions to future policies. ${ }^{4}$

2. SHARE. Leaders should model what they ask of frontline 
hospitalists and share their own feelings, even if they are experiencing mixed or negative emotions. For instance, a leader who is feeling saddened about the death of a patient can begin a meeting by sharing this sentiment. By allowing themselves to display vulnerability, leaders demonstrate courage and promote a culture of openness, honesty, and mutual trust.

3. INITIATE. Leaders should embrace difficult conversations and be transparent about uncertainty, although they may not have the answers and may need to take local responsibility for consequences of decisions made externally, such as those made by the health system or government. Confronting difficult discussions and being transparent about "unknowns" provides acknowledgement, reassurance, and shared experience that expresses to the hospitalist group that, while the future may be unsettled, they will face it together.

4. ANTICIPATE. Leaders should anticipate the emotional responses to operational changes while designing them and rolling them out. While negative emotions may heavily outweigh positive emotions in times of crisis, we have also found that harnessing positive emotions when designing operational initiatives can assist with successful implementation. For example, by surveying our hospitalists, we found that many felt enthusiastic about caring for patients with COVID-19, curious about new skill sets, and passionate about helping in a time of crisis. By generating a list of these hospitalists up front, we were able to preferentially staff COVID-19 teams with providers who were eager to care for those patients and, thereby, minimize anxiety among those who were more apprehensive.

5. ENCOURAGE. Leaders should provide time and space (including virtually) for hospitalists to discuss their emotions. ${ }^{8}$ We found that creating multiple layers of opportunity for expression allows for engagement with a wider range of hospitalists, some of whom may be reluctant to share feelings openly or to a group, whereas others may enjoy the opportunity to reveal their feelings publicly. These varied venues for emotional expression may range from brief individual check-ins to open "office hours" to dedicated meetings such as "Hospitalist Town Halls." For instance, spending the first few minutes of a meeting with a smaller group by encouraging each participant to share something personal can build community and mutual understanding, as well as cue leaders in to where participants may be on the emotional landscape.

6. NURTURE. Beyond inviting the expression of emotions, leaders should ensure that hospitalists have access to more formal systems of support, especially for hospitalists who may be experiencing more intense negative emotions. Support may be provided through unit- or team-based debriefing sessions, health-system sponsored support programs, or individual counseling sessions. ${ }^{4,8}$

7. APPRECIATE. Leaders should deliberately foster gratitude by sincerely and frequently expressing their appreciation. Because expressing gratitude builds resiliency, ${ }^{9}$ cultivating a culture of gratitude may bolster resilience in the entire hospital medicine group. Opportunities for thankfulness abound as hospitalists volunteer for extra shifts, cover for ill colleagues, participate in new working groups and task forces, and sacrifice their personal safety on the front lines. We often incorporate statements of appreciation into one-on-one conversations with hospitalists, during operational and divisional meetings, and in email. We also built gratitude expressions into the daily work on the Respiratory Isolation Unit at our hospital via daily interdisciplinary huddles for frontline providers to share their experiences and emotions. During huddles, providers are asked to pair negative emotions with suggestions for improvement and to share a moment of gratitude. This helps to engender a spirit of camaraderie, shared mission, and collective optimism.

\section{CONCLUSION}

Hospitalists are experiencing a wide range of emotions related to the COVID-19 pandemic. Hospital medicine leaders must have strategies to understand the emotions providers are experiencing. Being aware of and acknowledging these emotions up front can help leaders plan and implement the operational changes necessary to manage the crisis. Because our health system and city have fortunately been spared the worst of the pandemic so far without large volumes of patients with COVID-19, we recognize that the strategies above may be challenging for leaders in overwhelmed health systems. However, we hope that leaders at all levels can apply the lessons we have learned: to ask hospitalists how they are feeling, share their own feelings, initiate difficult conversations when needed, anticipate the emotional effects of operational changes, encourage expressions of emotion in multiple venues, nurture hospitalists who need more formal support, and appreciate frontline hospitalists. While the emotional needs of hospitalists will undoubtedly change over time as the pandemic evolves, we suspect that these strategies will continue to be important over the coming weeks, months, and longer as we settle into the postpandemic world.

Disclosures: The authors report having no conflicts of interest to disclose.

\section{References}

1. Chopra V, Toner E, Waldhorn R, Washer L. How should U.S. hospitals prepare for coronavirus disease 2019 (COVID-19)? Ann Intern Med. 2020;172(9): 621-622. https://doi.org/10.7326/m20-0907

2. Garg M, Wray CM. Hospital medicine management in the time of COVID-19: preparing for a sprint and a marathon. J Hosp Med. 2020;15(5):305-307. https://doi.org/10.12788/jhm.3427

3. Hertling M. Ten tips for a crisis: lessons from a soldier. J Hosp Med. 2020;15(5):275-276. https://doi.org/10.12788/jhm.3424

4. Shanafelt T, Ripp J, Trockel M. Understanding and addressing sources of anxiety among health care professionals during the COVID-19 pandemic. JAMA. Published online April 7, 2020. https://doi.org/10.1001/jama.2020.5893

5. Mintz LJ, Stoller JK. A systematic review of physician leadership and emotional intelligence. J Grad Med Educ. 2014;6(1):21-31. https://doi.org/10.4300/ jgme-d-13-00012.1

6. Goleman D, Boyatzis R. Emotional intelligence has 12 elements. Which do you need to work on? Harvard Business Review. February 6, 2017. Accessed April 16, 2020. https://hbr.org/2017/02/emotional-intelligence-has-12 -elements-which-do-you-need-to-work-on

7. Salovey P, Mayer JD. Emotional intelligence. Imagin Cogn Pers. 1990;9(3):185211. https://doi.org/10.2190/DUGG-P24E-52WK-6CDG

8. Kisely S, Warren N, McMahon L, Dalais C, Henry I, Siskind D. Occurrence, prevention, and management of the psychological effects of emerging virus outbreaks on healthcare workers: rapid review and meta-analysis. BMJ. 2020;369:m1642. https://doi.org/10.1136/bmj.m1642

9. Kopans D. How to evaluate, manage, and strengthen your resilience. Harvard Business Review. June 14, 2016. Accessed April 21, 2020. https://hbr. org/2016/06/how-to-evaluate-manage-and-strengthen-your-resilience 